\title{
SISTEMA Y MÉTODO DE LA PSICOLOGIA
}

\section{Confusión y desarrollo}

A pesar de que la psicología es probablemente la más nueva de las ciencias. que se ocupan de estudiar al hombre -entendiéndola precisamente como ciencia- cuenta a la fecha con una bibliografía tan abundante, a la cual seguramente no iguala en variedad de posturas ni abundancia de tratamientos, ninguna otra de las disciplinas que han prosperado con acelarado ritmo en el presente siglo; alberga dicha bibliografía una verdadera catarata de trabajos que enriquecen continuamente el saber psicológico y enaitecen la noble preocupación del ser humano en la ingente inquietud por conocerse a sí mismo.

Este conocimiento es tanto más urgente cuanto mayor es la evolución del hombre, teniendo en cuenta el gran número de problemas que han surgido por doquier a consecuencia de su creciente complejidad individual y social, aunada al natural deseo por afrontar de lleno su propio conocimiento.

Esta laudable inquietud, que proviene cuando menos de la antigüedad clásica, pretende ser resuelta ahora con la documentada labor de investigación que acometen los psicólogos, pertrechados con mejores armas que nunca, para abordar la sugestiva tarea de llegar a los rincones ignotos de la existencia y desentrañar los recónditos secretos de la personalidad.

Junto a este innegable hecho que se revela en la constitución de la psicologia moderna, es justo reconocer la abundancia de tratamientos que se le dedicaron desde la antiguiedad, cuando las ideas sobre el alma estaban incluidas en la mitología, en la religión, en la filosofía o las observaciones precursoras de la psicologia cientifica.

En la época moderna se amparan en el vasto tratamiento metodológico que en poco más de un siglo ha desarrollado multitud de problemas, encubiertos que se encontraban por el engañoso velo de la superstición o por el sugerente manto de la especulación, quedando durante siglos a espaldas dela cientificidad.

En el breve lapso que comprende aproximadamente una centuria, la psicología logró recuperarse del lamentable atraso en que había permanecido durante los veinticinco siglos de su existencia como psicologia precientifica (v. a. c.-xix d. c.), en los cuales se consagró principalmente a recoger creencias míticas, religiosas, metafísicas, filosóficas y empíricas, que tradujeron la multiforme inquietud del hombre por conocerse a sí mismo. Fue hasta mediados del siglo xIx cuando descubriéronse las motivaciones positivas que determinan la conducta del ser humano y se fijaron las normas metodológicas. para su tratamiento objetivo y progresivo. 
En este corto lapso, el desarrollo de la psicologia cientifica ha sido torrencialmente pródigo, como se comprueba en el creciente número de personas y escuelas que se dedican profesionalmente a ella, y en la cada vez más abundante bibliografía que recoge las excelencias de sus mejores frutos.

Tan vertiginoso desarrollo se ha traducido en un acopio de datos y teorías que cubren básicamente todo lo que pueda exponerse en relación a sus problemas, de acuerdo con el estado actual de la investigación; se ha profundizado en los temas de la psicología clásica que se refieren a las "facultades y la naturaleza del alma"; se ha afinado considerablemente el método introspectivo, que sirve de instrumento a gran parte de la psicología concreta, cuyo conocimiento del Yo resulta esencial para su desarrollo.

También se han puesto de lado - cuando menos en el dominio de la ciencia- multitud de prejuicios y supercherías que aherrojaban al conocimiento psicológico en los grilletes de la ignorancia y el fanatismo; se han determinado experimentalmente multitud de fenómenos psicobiológicos que permanecían inexplicados para la indoctrinación científica; se han tratado minuciosamente, en fin, las cuestiones dinámicas del psicoanálisis, en su proyección autónoma, biológica y cultural, gracias a la detenida exploración del subconsciente, que penetra en las facetas individual y colectiva del comportamiento, produciendo un nuevo ángulo de comprensión objetiva para la humanidad. También se han desenvuelto con amplitud las relaciones de la psicología con las ciencias afines, indagando en conjunto cuál es la naturaleza y conducta del ser humano.

Así tenemos un gran número de conocimientos obtenidos por las siguientes relaciones de la psicología con varias ciencias afines:

a) Psicología y sociología.

b) Psicología y antropología.

c) Psicología y biología.

d) Psicología y física.

e) Psicología y filosofía.

f) Psicología e historia.

Las anteriores relaciones concurren al común interés del hecho psicológico y aportan en cada caso los conocimientos obtenidos en su respectiva demarcación.

En estas condiciones, la gran cantidad de temas que maneja actualmente la psicologia cientifica, abre el mayor horizonte para desarrollar un estudio sistemático de la propia disciplina, estudio tanto más difícil de producir por cuanto reclama simultáneamente la documentación en los temas específicos y en el conjunto integral de las ciencias del hombre. Ellas forman el territorio más amplio y en cierto modo el más valioso de todos los que pueda pro- 
ducir el intelecto, al considerar que, en mayor o menor grado, toda ciencia admite una jurisdicción aplicable al conocimiento del hombre.

Sobre esta perspectiva nos proponemos efectuar una sucinta exposición de los principales temas psicológicos, observando los motivos de cada uno para extraer su problemática general y ensayar un examen comparativo de sus diferentes direcciones, el cual nos permitirá concluir en la importancia que pueda tener cada una para el conocimiento del hombre, situadas como se encuentran en el complejo panorama actual de tan importante disciplina.

\section{Pluralidad doctrinaria}

El material que incluimos en este artículo está constituido por la observación esencial en las principales vertientes que alimentan a esa ciencia, que en última instancia es solamente una, pues sus diversos temas corresponden al problema básico que se expresa en la siguiente pregunta: ¿Qué es el hombre? O para precisar mejor: ¿Qué es la conciencia del hombre? Los tratamientos básicos que origina semejante problema se han desenvuelto hasta un grado suficiente para considerarlos como formas autónomas y específicas de la psicología.

Por ello se puede hablar en cierto modo de psicologia y psicologias, siempre y cuando se mantenga en ellas una relación similar a la que existe - por ejemplo- entre la física y las diferentes ramas de la física; así como en la ciencia natural un capítulo de mecánica figura junto a otro de óptica, otro más de termodinámica, uno de hidrostática, etcétera, así pueden aparecer, con la misma unidad y congruencia, las distintas ramas que constituyen a la psicologia y que habitualmente se les toma como escuelas, posturas, direcciones o ciencias psicológicas distintas, cuando no contrapuestas.

El primer problema que plantea el estudio de esta ciencia consiste en ofrecer una correcta definición de la misma, pues de lo contrario se cae en el inevitable riesgo de extraviar sus problemas, esfumar sus contornos y confundir sus procedimientos. Tal es lo que acontece realmente en la mayoría de los casos, pues, como hemos dicho, la psicología registra tamaña variedad de acepciones que resulta difícil caracterizarla en forma univoca.

Observada históricamente, priva de una época a otra divergencia en problemas y métodos, que el nombre de psicologia viene a quedar casi en calidad de un mero nombre, al punto que inclusive el profesional suele tener alguna dificultad para orientarse en la intrincada muchedumbre de prospectos que surgen al paso de su estudio.

La mayor dificultad para abarcar el panorama psicológico se encuentra en la radical especialización que asiste a la propia psicología en sus diferentes posturas, las cuales han llegado a diferir profundamente. El psiquismo es para unos todavía la entidad inmaterial de que nos habla la metafísica, mien- 
tras que para otros representa una realidad concreta y material, asequible por medio de la experimentación; la constitución psicológica está, según aquéllos, fatalmente predeterminada por la naturaleza y el hombre nada podrá hacer para modificarla, mientras éstos la conceptúan como una entidad moldeable, inmersa en la experiencia y susceptible de modificarse notablemente con el impulso formativo de la educación.

Entre ambas posiciones, la que sostiene al "alma" como una entidad metafísica, abstracta y predeterminada, y la que afirma su concreción, materialidad y evolutividad, se encuentran un gran número de matices intermedios que representan sendas etapas en el desarrollo histórico de la psicología. Es radical la diferencia entre el tratamiento que suele concederse a esta disciplina en la corriente tradicional y el que se le otorga en sus modernas direcciones.

Podríamos citar inclusive al tipo de acontecimientos cuyo extraordinario carácter ha hecho pensar que se trataría de fenómenos "sobrenaturales"; también ellos ocupan actualmente la atención de la psicología, en la rama denominada parapsicologia o metapsíquica, diversificada como se encuentra en su gran preocupación por el estudio de tan sugerentes y variados acontecimientos.

La abundante presencia de las manifestaciones psicológicas explica la complejidad de esta apasionante disciplina. Pero el panorama se complica todavía más con las esferas de influencia que admiten los acontecimientos psíquicos; no se producen aislados sino en un estrecho vínculo que origina la compacta estructura donde se entrelazan las numerosas manifestaciones del espíritu. Es comprensible que ante esta marcada complejidad existan numerosas divergencias de criterio para la apreciación de sus métodos y procedimientos, de donde las variadas doctrinas que se encuentran a cada paso en su estudio.

El proceso histórico operado en el campo de la psicología es sensiblemente análogo al que observamos en las demás ciencias; tiene en común con ellas el denotar una creciente especialización en el tratamiento de sus cuestiones. Este hecho obedece a la elocuente circunstancia expuesta en el continuo aumento de los descubrimientos y problemas que va produciendo la ciencia, lo cual hace cada vez más difícil compenetrarse de ella y prácticamente imposible dominar satisfactoriamente toda su latitud disciplinaria.

El proceso de especialización en la ciencia puede expresarse diciendo que los sabios tienden a saber cada vez más sobre cada vez menos, es decir, a obtener un mayor número de conocimientos sobre una menor extensión de temas. Por ello, la especialización afecta notablemente a los estudios psicológicos, en cuanto determina la formación de especialistas que, en el mejor de los casos, conocen una sola ramificación de esta ciencia y, en el peor, no conocen a fondo ninguna. Ésta es obviamente una desventaja, por cuanto concierne a la ignorancia de los motivos que se soslayan o recaen fuera de su especializa- 
ción; pero también permite el gran avance proveniente de la especialización misma, o sea el impulso otorgado al saber mediante el dominio de los problemas que se plantean específicamente en cada una de las disciplinas y cada una de sus ramificaciones.

En virtud de la especialización, el conocimiento científico ha aumentado notablemente en los últimos años, pero el precio que por ello se paga es que los psicólogos -igual que todos los científicos modernos- conocen cada vez más de su propia especialidad y cada vez menos de las demás.

\section{Problemas y especialidades}

De lo que acabamos de ver se advierte que el estudio de la psicología es abordable mediante el examen de los grandes sistemas que se manifiestan como direcciones fundamentales de la psicología, así como también las escuelas y posturas particulares que derivan de cada dirección, por efecto de la correspondiente particularidad de sus problemas.

Este sistema de estudio se encuentra en todas las disciplinas psicológicas, pues cada una produce una diferente especialización del saber, que parte, sin embargo, del planteamiento señalado por una pregunta de carácter general: ¿Qué es y cómo funciona la conciencia humana?

A partir de este planteamiento se originan varias especializaciones que corresponden a las diferentes maneras de concebir y afocar el problema del ser consciente, el cual figura como denominador común en todas las proyecciones de la psicología científica. Por otra parte, la evolución de las grandes direcciones psicológicas ha dado origen a sendos capítulos esenciales y constitutivos de esta disciplina, lo cual obedece a la presentación de otros tantos problemas capitales, que corresponden a los consiguientes aspectos del objeto primordial que es abordado por el estudio cientifico de la psicología.

Así tenemos que esta relación de carácter metodológico establecida entre cada uno de los grandes problemas y la correspondiente especialidad del saber, explica la génesis de las diversas posturas psicológicas, demostrando cómo se originan en los aspectos parciales derivados del problema esencial que se debate en la propia disciplina, es decir, corresponden a maneras diferentes de plantear el problema de la conciencia.

A su vez, cada una de las grandes ramas psicológicas da cauce a nuevas especializaciones y subespecializaciones que alcanzan un nivel de mayor particularidad a medida que el conocimiento avanza en el estudio de los fenómenos concretos, percibiendo directamente la realidad del problema psicológico que plantea la consideración del aspecto respectivo.

He aquí, pues, los puntos concluyentes en la observación preliminar que estamos efectuando. Existen en la psicologia -igual que en toda cienciatres dimensiones que definen su planteamiento básico: 
a) Problema esencial. Es el que corresponde en común a todos los problemas y tratamientos específicos que asume una disciplina científica, en este caso la psicología.

b) Aspectos generales. Son las diferentes maneras que tiene el problema psicológico de manifestarse a la consideración de las grandes direcciones o escuelas que abordan su tratamiento.

c) Especialización. Es el tratamiento de los problemas específicos que se manifiestan en un creciente nivel de particularidad, a medida que se aproximan a los hechos concretos.

Esta integración del saber exhíbese en todas las disciplinas científicas, que precisamente por serlo dan origen al variable planteamiento de los problemas y la correspondiente resolución por conducto de los métodos y sistemas con los cuales se construye el cuerpo doctrinario que corresponde a la psicología. De manera recíproca, la creciente especialización de las investigaciones, a partir del planteamiento general, situado en el conocimiento de la conciencia humana, da origen a la disposición arborescente, o sea de una.continua especialización, que representa el sistema de acción y desarrollo en cada disciplina.

Podríamos mencionar, a título de ejemplo, cualquiera de las grandes ciencias que produce el saber humano - la física, la biología, la sociología, etc.-, pero estando ya situados en el estudio de la psicología, nada mejor que dirigirnos a ella para comprobar lo que acabamos de señalar en relación al saber científico, observado en sus aspectos general y particular.

El problema de la psicología no constituye una salvedad a la evolución dinámica del pensamiento; por el contrario, es uno de los casos más evidentes donde se aprecia la disposición que adquiere el sistema integrativo de acuerdo con la creciente especialización que asumen las investigaciones. Así tenemos que, por principio de cuentas, existe un tema general de toda la psicología y obedece a una misma realidad, como es la relativa al funcionamiento interconsciente del psiquismo. Sobre lo que sea en última instancia la conciencia o el psiquismo, existen multitud de teorías, desde las que considéranla como una función biológica y material, hasta las que proclaman en ella los caracteres más decantados de espiritualidad.

Sin embargo, priva un acuerdo básico y consiste en afirmar la existencia del psiquismo, representado fundamentalmente por la conciencia, que a su vez se manifiesta en la conducta, sobre lo cual están de acuerdo todas las posturas psicológicas. En efecto, cada uno de nosotros percibe el funcionamiento de la conciencia como la manifestación más elevada de su propia naturaleza, y más allá de los desacuerdos que pueden aparecer como criterio distintivo de las posturas psicológicas, se encuentra el unánime consenso de que existe el psiquismo, y constituye la expresión más elevada y representativa del hombre. 
A partir de este principio fundamental se erige el problema de la psicologia básica como ciencia explicativa de los fenómenos psíquicos. La totalidad de las doctrinas psicológicas está de acuerdo en reconocer que la manifestación fenoménica del psiquismo cifra en torno a la conciencia, cuya expresión es la más genuinamente humana, debido a lo cual se considera a la psicología, explícita o implícitamente, como ciencia de la conciencia.

El horizonte psicológico se extiende constantemente con el tratamiento de algunos aspectos humanos que colindan con la conciencia, pero no se identifican con ella; así tenemos, de manera principal, los diferentes factores biofisiológicos que estudia la psicología biológica o naturalista; también el recién descubierto tratamiento del inconsciente, que se revela en el psicoanálisis, cuyo sistema corresponde a la psicología analítica; finalmente, la interacción de los individuos que tiene lugar en el seno de la colectividad, de lo cual se ocupa la psicologia social.

Así tenemos perfiladas las grandes orientaciones de la psicología, que corresponden a sendos planteamientos en el problema de la conciencia:

a) Psicologia básica. Es el sistema que reconoce la conciencia individual y toma como punto de referencia el ejercicio normal de sus facultades.

b) Psicología biológica. Es el sistema que reconoce la acción del inconsciente como definitivo en la actividad normal y anormal de la conciencia.

c) Psicologia social. Es el sistema que investiga las relaciones entre la conciencia individual y el comportamiento colectivo, partiendo de su interacción recíproca.

Digamos ahora algunas palabras sobre cada una de estas direcciones fundamentales de la psicologia.

\section{Las cuatro direcciones}

El punto de partida que adoptamos para apoyar la definición de la psicología como ciencia de la conciencia, es el que se contiene en el planteamiento de los problemas que tradicionalmente maneja. En efecto, la definición básica de psicología tiene como punto de referencia las diversas formas que asume la conciencia y los grandes problemas que determina. La psicología tradicional se dirige básicamente al contorno fenoménico de la conciencia, tal como es percibida introspectivamente por el sujeto en el propio Yo. Las nociones que le proporciona esta observación complementan las referencias indirectas que obtiene de los demás, llegando a un cierto grado de objetivación en sus conocimientos mediante la reiterada frecuencia del examen que efectúa dentro y fuera de sí mismo, lo cual le induce a concebir la estructura esencial que constituye la base para edificar el sistema psicológico.

Este planteamiento hizo posible la llamada psicología clásica, tradicional o académica, que designamos también como psicología básica, cuya fuente de 
documentación es predominantemente introspectiva, pues se dirige a la manifestación de la conciencia tal como es percibida por el sujeto.

También se le llama clásica, pues su extensión histórica a través de casi veinticinco siglos de existencia, convirtiola en una tradición clasicista que fue impartida durante largo tiempo como enseñanza académica. Y básica la llamamos nosotros, porque es fruto de una observación que el sujeto efectúa en sí mismo para inducir de ahí las generalidades a que le constriñe la realidad psicológica.

La segunda de las grandes vertientes psicológicas está constituida por otro de los aspectos fundamentales, a saber: el análisis del psiquismo en el plano representado por la ciencia natural. . Este punto de vista corresponde a la constitución biológica que posee el hombre, desde el momento que existe en un organismo físico y sirve de sustento a la organización psíquica, la cual resulta - según los más denodados defensores de esa postura- por completo asimilable a la estructura material. De acuerdo con ella, el psiquismo se reduciría únicamente al nivel superior de la organización biológica expresada por la manifestación consciente del hombre.

Quienes defienden la tesis de que el ser humano es en todos sus aspectos una entidad material, han desplegado esfuerzos para comprobar esa teoría mediante un estudio cada vez más intenso y penetrante de los fenómenos biológicos que acompañan a la actividad psíquica; o mejor dicho, de los fenómenos que constituyen dicha actividad, pues tal es la interpretación que debe otorgársele, para ser fieles a la radical postura materialista que expone semejante teoría.

Como ese aspecto material se manifiesta a través de la organización biológica, resulta de ahí que la psicología naturalista y materialista se erige como una psicologia biológica, cuyo basamento científico está apoyado por las numerosas ciencias en que se funda, e integran en conjunto el sistema de las ciencias naturales.

Así acuden en apoyo de la psicología materialista, no solamente la biología general, sino específicamente las ciencias médicas y antropológicas; también el vasto complejo de las ciencias físicas, e inclusive las matemáticas adquieren una poderosa intervención en el desarrollo de esta psicología, porque la calidad de ser vivo que posee el hombre es el carácter que lo sitúa en la relación genérica con las ciencias que se ocupan de estudiar a la naturaleza, matizando inclusive la acción de la conciencia con un elevado coeficiente material, de donde la motivación predominante en esta psicología es la orientación biológica.

Sus partidarios gustan de llamarla cientifica, porque propugna el estudio experimental de los fenómenos psíquicos, como si se tratara de cuestiones netamente materiales, asequibles por medio del laboratorio.

Reconoce también la atribución básica de la psicología como ciencia de 
la conciencia, desde el momento en que su actividad se constituye como eje de experimentaciones, las cuales tienen efecto sobre el aspecto material del psiquismo, expuesto como se encuentra en el hecho innegable de que la acción consciente del individuo se produce en el seno de la persona física, que representa la realidad material del ser humano.

La tercera de las grandes corrientes que señalaremos es probablemente la más nueva y al mismo tiempo la que más se ha desarrollado en los últimos tiempos. Se trata de la psicología analitica, cuyo fundador es Sigmund Freud, quien realizó las primeras investigaciones sistemáticas tendientes a fincar en forma definitiva los cimientos de esta nueva orientación.

Su punto de partida consiste - como todo el mundo sabe- en el redescubrimiento del inconsciente y su tratamiento dinámico en relación a los problemas de la conciencia. Aunque antes de Freud se habia señalado que existe esa importantísima esfera de la personalidad —el inconsciente- Freud llevó a cabo su primera exploración profunda, incidiendo en los numerosos aspectos y problemas que reviste la afirmación del inconsciente como una fuente de conducta que asume trascendental importancia en la personalidad.

Los variados problemas que implica la acción inconsciente sobre la conciencia y la conducta, fueron explorados por Freud en el terreno de la psicopatologia, es decir, en el campo de las observaciones que tienen como objeto de estudio a los seres que, por padecer algún desequilibrio psicológico, se consideran como enfermos o anormales. El descubrimiento freudiano consistió en demostrar que la acción del inconsciente no se limita a un papel meramente pasivo, como se había creído con anterioridad; se sabe ahora que las motivaciones inconscientes obedecen a un importante acervo de experiencias que ejercen definitivo influjo en la personalidad.

El carácter analítico de esta psicología concierne primordialmente a su método, consistente en analizar la organización psíquica y escindirla en sus componentes primarios, para lo cual requiere ir más allá de la manifestación aparente de la personalidad e ingresar en su constitución profunda. De ahí el calificativo de analitica que recibe esta psicología, así como también el método que emplea para su desarrollo, universalmente aceptado con el nombre que le otorgó su fundador: psicoanálisis.

El aspecto más importante de dicha postura radica en la modalidad específica que consiste en el análisis del inconsciente, planteado en un principio por la investigación freudiana y seguido con posterioridad por un sinnúmero de prosélitos. El acuerdo existente en todos ellos - por encima de las numerosas discrepancias que también acusan- radica en aceptar a la organización psicológica como una estructura dinámica, integrada por dos estratos fundamentales, que corresponden al inconsciente, o sea la zona de la personalidad de la cual el individuo no es consciente, y la conciencia propiamente dicha, como es el conjunto de elementos que configuran el decurso efectivo del exis- 
tir, donde la deliberación racional y volitiva asume una importancia de primer orden.

Llegamos al cuarto y último de los aspectos que se revelan en el psiquismo, abordando el tema que probablemente ha sido menos tratado que los demás. Tal es la psicología social, que, como su nombre lo indica, constituye una aplicación del problema psicológico a la organización colectiva, donde se manifiesta una forma de comportamiento que revela la existencia, directa o indirecta, de una conciencia masiva.

La psicología social se pregunta a qué normas obedece el comportamiento de los pueblos y qué relación puede establecerse entre la conciencia individual y la conciencia colectiva. Ambas cuestiones requieren un amplisimo volumen de investigaciones para contestarlas satisfactoriamente; el problema se agudiza todavía más, porque el comportamiento de los pueblos recae dentro de la evolución histórica, o sea que no existen dos sociedades y, por consiguiente, dos formas de comportamiento absolutamente iguales.

Cada época y cada lugar señalan un caso especifico de colectividad, cuya conducta puede y debe ser investigada atendiendo a los caracteres singulares que presenta en su condición única e irrepetible. Las sociedades históricamente producidas adquieren un perfil que exhibe los mayores rasgos individuales, a pesar de lo cual el problema de esta psicología subsiste en los términos de generalidad que hemos dicho.

La psicología social recibe un auxilio poderoso de la antropología, de la historia, la filosofía y otras ciencias que coadyuvan eficazmente a la resolución de su problema, el cual se plantea en dos preguntas como las siguientes: ¿Qué condiciones generales explican el comportamiento colectivo? ¿Qué relación existe entre la conciencia individual y la conciencia colectiva?

Digamos, por último, que para resolver esta cuestión los psicólogos se abstraen de la realidad individual para dirigirse a la entidad superlativa donde conviven los hombres, que se denomina sociedad. Quienes cifran el estudio psicológico en la realidad social, aceptan la existencia de la conciencia individual como punto de partida en sus consideraciones de sociopsicologia, pero tienen como necesaria referencia el reconocimiento de la múltiple acción diferencial que se traduce en la conciencia colectiva.

Por ello, los psicólogos de la sociedad nos hablan de una conciencia social, que no contraponen a la individual, sino que la presentan como derivación o consecuencia de ésta, pero cuyos caracteres se exhiben con amplia y modificada dimensión en los fenómenos de orden colectivo.

\section{La psicología sincrética}

Para terminar esta sección, explicaremos cuál es el significado que entraña la psicología sincrética, en cuyo concepto se funda la perspectiva que recono- 
cemos en esta disciplina. Ante todo, el concepto de sincretismo representa el establecimiento de la unidad sistemática y estructural en un conjunto de opiniones divergentes que, sin embargo, se complementan en virtud de sus funciones integrativas, como son las que manifiesta el decurso evolutivo de la psicología en paralelo a la estructura unitaria del psiquismo.

El punto de vista capital que rige el desenvolvimiento de esta ciencia se localiza en una secuela consistente en el reconocimiento de diversos problemas psicológicos que dan origen a sendas posturas o direcciones de trabajo, de donde la necesidad de establecer un criterio capaz de comprender en sintesis integral las diferentes actitudes de investigación e interpretación asumidas frente al tema, cada una de las cuales tiene su propia razón de ser.

Por ello debemos buscar, en último término, la construcción de un amplio sistema que reúna los diversos planteamientos del temario psicológico en una doctrina sincrética. La necesidad de semejante sistema se manifiesta al comparar las diversas doctrinas que se han producido, observándolas desde un plano superior a cualquiera de ellas, trascendental con respecto a las posiciones particulares que asume el estudio psicológico en virtud de la creciente especialización a que nos hemos referido y cuya consecuencia suele consistir en la incomprensión de cada especialista frente a las otras posiciones, así como el estéril antagonismo que se produce en las diferentes escuelas y la invasión recíproca de sus problemas, que al afocarse unilateralmente omiten el correlativo desempeño que atañe a las otras facetas de la personalidad.

Abarcando las cuatro grandes direcciones que señalamos, recordemos que cada una contiene un punto de vista específico, una razón de ser en cuya virtud es admitida por un gran número de tratadistas que se ocupan de sus problemas privativos. Observando en perspectiva las cuatro direcciones capitales, salta a la vista la necesidad que originó a cada una y que comentaremos brevemente.

En primer término, la psicología básica responde a que en ella se originan los problemas fundamentales de esta ciencia, revelados en la observación directa del psiquismo. En esa dirección se establecen las nociones básicas que todavía se emplean para exponer el $\mathrm{ABC}$ de las consideraciones psicológicas, y que a pesar de las numerosas impugnaciones dirigidas en su contra, al amparo de la evolución experimentada por la psicología moderna, podemos afirmar que no se destruye, sino que, al contrario, se complementa con la investigación actual, integrando un magno edificio del que la psicología básica constituye su fundamento, mientras los demás capítulos equivalen a la edificación de sus niveles constructivos.

Por su parte, la psicología biológica encuentra razón de ser en los coeficientes de orden material que actúan en la realidad constitutiva del ser humano, y que no es posible soslayar, pese a los numerosos intentos que a tal 
respecto se han efectuado en el campo de la psicología espiritualista. El conocimiento de la personalidad debe tener en cuenta esta determinación biológica; los invaluables estudios que han llevado a cabo multitud de cientificos en los últimos años, revelan que la estructura biofisiológica expone una creciente determinabilidad que indica cuál es la constitución real y natural del ser humano.

Por su parte, la psicologia analítica demuestra que la conducta está regida por una mecánica interpsíquica cuyo basamento es el inconsciente; ahí afloran numerosas motivaciones recónditas que, sin embargo, se manifiestan poderosamente -aunque disfrazadas - en el comportamiento. Esta doctrina cuenta con el poderoso recurso metódico que significa el psicoanálisis, cuya aplicación al tratamiento de los casos psicopatológicos se ha extendido en forma sorprendente durante el último medio siglo.

Por fin, la psicologia social nos recuerda que el ser humano nace y se desenvuelve en la colectividad, considerada tanto en el aspecto genérico de la nación a que pertenece, como en los diversos tipos de comunidades que produce la convivencia humana. Gran parte de los atributos y también de los padecimientos que caracterizan al hombre moderno, se originan en la convivencia social, que adquiere cada día mayores caracteres definidos, normales y patológicos, de suerte que no es posible plantear válidamente el problema del psiquismo si esta relación intersocial es omitida.

Como puede observarse, la participación de las cuatro grandes vertientes resulta indispensable para el planteamiento integral de la psicología. A pesar de ello, la actitud que encontramos generalmente en el campo de la psicología profesional, suele no corresponder a esta necesidad de integración sistemática; es frecuente escuchar a los más connotados especialistas opinando que su propio sistema es el único válido, por lo cual no conceden suficiente beligerancia $\longrightarrow$ tal vez ninguna- a los demás.

Por ello existen todavía los tradicionalistas que se aferran a las nociones elementales de la psicología básica y desearían inclusive presentarla como una rama de la filosofía, sin admitir los inconmensurables progresos que ha efectuado como ciencia autónoma. Otros hay que se empeñan tenazmente en reducir su problemática a experimentos de carácter mensural, y la imagen que en ellos se produce corresponde a una inaceptable disgregación empiriológica de la conciencia. También los fanáticos de la psicología analítica lanzan un intolerante cuarto a espadas al pretender que el psicoanálisis constituye una fuente absoluta de interpretación, panacea para los trastornos de la conciencia. Por último, los partidarios radicales de la psicologia social no se quedan a la zaga y desean que el individuo se convierta en una simple célula del vasto organismo que es la sociedad, restándole por completo la autonomía inherente a su calidad individual.

Como resultado de esta disención que produce la estrechez de miras en 
cada una de las posturas específicas, se manifiesta el antagonismo y la incomprensión que privan en gran parte del campo profesional de la psicología. Para superar tales deficiencias es necesario esgrimir el punto de vista sincrético que - según hemos dicho- consiste en tomar en cuenta todos los planteamientos objetivos que se producen en torno al problema psicológico, partiendo de los diversos aspectos que lo constituyen; de esta suerte, cada uno adquiere su propia autonomía que le permite subsistir con independencia de los demás, sin que ello sea motivo para oponerse a los designios ajenos.

Este sincretismo psicológico sólo puede erigirse una vez que las distintas especialidades derivadas de cada planteamiento específico han sido suficientemente comprendidas en su propio ámbito, con la necesaria ductilidad para reconocer la validez de los puntos de vista que asisten a las demás posturas.

De aqui podemos concluir nuestras meditaciones afirmando que la psicología sincrética representa la unidad estructural de las doctrinas psicológicas, que resultan aceptables siempre y cuando se apoyen en un criterio estricto de objetividad. Empero, ciertas ideas precientíficas que pertenecen a la filosofía y la religión pueden inclusive ser comprendidas a título de estadios precursores, e incluirse históricamente como una concepción anticipada de la psicología, sin olvidar que la psicología científica es, en última instancia, la única que puede y debe aceptarse en la actualidad.

\section{Unidad de las posturas}

La exposición que efectuamos de las diversas proyecciones que asume la psicología a través de su integración histórica, distinguiendo fundamentalmente cuatro grandes posturas que son la psicología básica, biológica, analitica y social, constituye una prueba elocuente de la versatilidad que adquieren sus escuelas doctrinarias, con la correspondiente ductilidad que debe existir en el criterio para su comprensión.

Por ello, frente a la variedad de teorías psicológicas no debe adoptarse una actitud rígida que quisiera mantener una sola y rechazar a las demás, sino una comprensión dúctil, que explique el origen de las variadas doctrinas obedeciendo a los diferentes aspectos del psiquismo que se manifiestan en la realidad y que a su vez originan los problemas integrativos en el estudio del ser humano.

La actitud contraria consiste en asumir una posición unilateral e invariable, que corresponde por regla general al concepto que profesa cada quien del psiquismo, derivado a su vez de una cierta especialización característica de nuestra época, pues la extensión del estudio psicológico y antropológico en general, se ha difundido a todas las ramas del saber, a tal punto que resulta difícil avanzar en cualquiera de ellas sin especializarse en el sistema respectivo. De ahí surgió el creciente particularismo que exhiben los psicólogos 
profesionales que, por regla general, se ubican en un solo sistema y por consiguiente suelen ignorar a los demás.

Lo que hemos dicho respecto a las posturas psicológicas puede también afirmarse en relación a los métodos. Existe una marcada tendencia a adoptar un solo método de trabajo, correspondiente a la postura asumida, que a su vez depende del problema planteado en torno a la conducta humana. Así tenemos una secuencia de la investigación psicológica donde se eslabonan los siguientes conceptos:

$$
\text { Aspectos } \rightarrow \text { Problemas } \rightarrow \text { Métodos } \rightarrow \text { Sistemas. }
$$

Ya explicamos que, para nosotros, cada uno de los grandes problemas psicológicos recoge una forma de manifestarse el psiquismo y el hecho de reconocer alguna no autoriza expresamente a negar a las demás, de análoga manera a como la existencia en el cuerpo humano de un aparato respiratorio - por ejemplo- no autoriza a denegar el aparato digestivo, circulatorio o nervioso, ni tampoco a pretender una explicación exhaustiva de la fisiología, limitándose exclusivamente a observar cualquiera de ellos.

El sistema biológico y el psicológico están integrados por una diversidad de elementos cuya participación es indispensable para asegurar su buen funcionamiento, de donde cualquier suposición que tienda a aislar uno o varios de dichos sistemas, resultará infundada y contraproducente. Por ello, al ingresar en nuestro examen de los sistemas psicológicos, señalamos la estrecha relación que existe entre la postura asumida frente al problema, y del problema con respecto al método. Esta relación se observa no sólo en psicología, sino en cualquiera otra ciencia.

De acuerdo con los conceptos que entraña dicha relación, el solo planteamiento de un problema implica el método adecuado para estudiarlo, pues en los términos del planteamiento está contenida potencialmente la resolución del mismo.

Por lo que hemos dicho, establecer la problemática psicológica es fundamental para esta disciplina; en efecto, el propósito que abrigamos para desarrollar de inmediato es la presentación sinóptica de la psicología, lo cual debe tener como punto de partida la verdadera comprensión de su problemática.

Para delinearla con alguna precisión vamos a señalar en primer término la existencia del problema psicológico mediante la caracterización general que hemos efectuado de la conciencia y la conducta, pues en ella radica el problema general de la psicología. Este problema es general, según decimos, porque se manifiesta en todas las formas de la conducta y participa asimismo en los diferentes planteamientos de la psicología.

Semejante problema general se traduce en varios problemas particulares que corresponden a las diversas formas de manifestarse la conducta; ya en- 
contramos que dichos planteamientos integran las grandes corrientes psicológicas, a saber: la psicología básica, biológica, analítica y social. En cada una se contiene un problema especifico y definido, según las circunstancias que en él influyen y lo determinan, correspondiendo - para seguir la misma clasificación- al problema básico, biológico, analítico y social de la psicología. Su caracterización respectiva puede ser la siguiente:

a) Problema básico. Estriba en la descripción de los elementos y procesos fundamentales de la conciencia.

b) Problemas biológicos. Radican en la determinabilidad de la conducta según los factores biológicos del psiquismo.

c) Problema analitico. Implica el análisis dinámico de la personalidad, observando la acción del inconsciente.

d) Problema social. Explica el comportamiento de acuerdo con los factores sociológicos que en él influyen.

Tales son los cuatro grandes problemas de la psicología, que corresponden a sendas modalidades del ser humano; cada una se manifiesta a través de la conducta, según el carácter específico que le es privativo. Por ello - repetimos- cada problema da origen a una postura psicológica que se manifiesta en el desarrollo de la investigación e integra la escuela correspondiente.

Recordemos que el concepto de método señala, en términos generales, un camino para resolver el problema planteado; éste; el problema, es precisamente una incógnita que demanda resolución. Podría parecer, y así se ha creído con frecuencia, que al implantar una diversidad de métodos pueden emplearse todos indiscriminadamente; pero no sucede así, pues el planteamiento correcto de un problema constriñe al empleo de un método adecuado. En otra forma se confunden y entremezclan nocivamente los procedimientos metódicos que deben seguirse para resolver los problemas que presenta el psiquismo.

De ahí que lo aseverado en relación a las posturas psicológicas se aplique directamente a la cuestión del método, según el problema que se registra en cada una de las diversas escuelas de la psicología. Todos los sistemas reconocidos estudian sendos aspectos de la conducta y dan origen a los problemas correspondientes, reclamando en cada caso el método adecuado para su resolución. Veamos, pues, cómo se constituyen los diversos métodos psicológicos a partir de la relación que establecemos.

Lo que hemos dicho en torno a los problemas de la psicología, puede aplicarse al método, pues la elección del método no es arbitraria, por el contrario, está determinada por el problema al cual se aplica, en virtud de la relación homogénea que debe existir entre problema y método. Para hacer factible la resolución del primero es necesario proseguir el camino representado por el segundo.

Así tenemos que la psicología básica implica su propio método, consis- 
tente en la observación directa de los fenómenos psíquicos, ya sea por la vía introspectiva, que atañe a las vivencias acaecidas en el sujeto que las observa, o bien mediante la extrospección, que se refiere a los acontecimientos sucedidos en el prójimo. En ambos casos se emplea un método que designamos como básico o directo, cuya dualidad de posibilidades obedece a la bifurcación que puede asumir el conocimiento directo de los hechos psicológicos: introspectivo o extrospectivo.

Por su parte, la psicología biológica emplea el método que corresponde a su problema, y como éste se erige en términos de realidad natural, el método a emplear será el naturalista. Dicho método examina los sucesos de la naturaleza conforme al método experimental, de donde se conoce también como experimental o naturalista. Consiste en manejar los elementos fisiológicos que participan en la vida psíquica de acuerdo con la relación de causa a efecto, determinando cuáles son las causas que producen ciertos efectos y, recíprocamente, de qué manera pueden explicarse los hechos como efecto de ciertas causas. Es obvio que ese método sostiene el carácter natural y material del psiquismo.

Por lo tocante a la psicologia analitica, su problema consiste en descubrir la acción dinámica del subconsciente, de donde el método radicará en descubrir tales elementos para explicar los actos de la conducta. Tal es el propósito que persigue el psicoanálisis, o sea el método de la psicología analítica; su desarrollo radica en promover un diálogo entre el psicólogo y el sujeto analizado, para explicar cuál es el origen de los conflictos que lo afectan.

Por último, el método de la psicología social corresponde también a su respectivo problema; tiene como punto de partida la observación del notable influjo que ejerce el medio ambiente en la formación del individuo. Por consecuencia, este método consiste en señalar cuáles son los coeficientes sociales que influyen mayormente en la conducta de los individuos, la forma como opera dicha influencia, así como los efectos que produce en las modalidades del comportamiento colectivo, o sea la tendencia que exhiben los grupos humanos para actuar según una forma análoga de conducta que corresponde a la idiosincrasia colectiva.

Así pues, para estudiar el método que emplea la psicología, es necesario advertir en primer término la diversidad de posibilidades metodológicas que se presentan, de acuerdo a las variadas posturas que derivan de los problemas localizados en el psiquismo y que a su vez corresponden a los diferentes aspectos en que se manifiesta.

No se puede afirmar que exista un solo método, un solo problema o una sola postura que pretendiera asimilar integramente la realidad psicológica, pues siendo ella variada y múltiple, da origen a los aspectos en que se mani- 
fiesta, los problemas que plantea, los métodos que se aplican a su desarrollo y las posturas que recogen las soluciones correspondientes.

De ahí que, para estudiar el método psicológico, deba seguirse un camino similar al que proseguimos en los problemas y las posturas. Así como llegamos entonces a la conclusión de que el camino es múltiple, y a pesar de todo se reconoce una cierta unidad al hablar del problema psicológico y de la psicología misma, así también es factible concluir en la existencia de un método que se integraría por la totalidad de los métodos parciales, pues cada uno desempeña una función específica. $\mathrm{Y}$ así como en el caso de las posturas. y problemas hablamos de una psicología sincrética que sintetiza y vincula a todas, así también se localiza un método sincrético que representa la unidad y la totalidad de los métodos parciales. Como base para determinar en qué consisten dichos métodos parciales en las posturas específicas que venimos sosteniendo, diremos algunas palabras sobre el que llamamos método básico, fundamental o primario en su estimación dinámica.

\section{El método básico}

El método que corresponde en general a la psicología básica, cuyo problema consiste en exponer las facultades o procesos generales del psiquismo, radica en explicar cómo se llega al conocimiento primordial de la conciencia. Dicho método procede a base de observaciones directas en la experiencia vital, sobre el flujo de la conciencia que acciona en indisoluble relación respecto a los: determinantes biológicos y las experiencias vividas, así como frente al medio que la envuelve y los individuos que integran la colectividad.

Ese método se lleva a cabo sobre dos grandes caminos que traducen sendas posibilidades de acción, según que las observaciones se efectúen en el sujeto mismo o se proyecten en el prójimo. En cada caso se habla generalmente de un método específico; para nosotros son dos modalidades del método que designamos como básico o esencial, y consiste en observar directamente la experiencia psíquica a través de los conductos perceptivos, produciendo las. acepciones precitadas, que consisten respectivamente en la observación de uno mismo, en cuyo caso es introspección, o de los demás, dando lugar entonces a la extrospección. Sus definiciones pueden ser las siguientes:

a) La introspección o introversión es el método que consiste en observar la experiencia psicológica en nosotros.

b) La extrospección o extroversión es el método que consiste en observar la experiencia psicológica en el prójimo.

La fase interiorizante conocida como introspección o introversión es el camino metódico insustituible para percibir el flujo vivencial que se desenvuelve en nosotros mismos; capta directamente los diversos motivos de su funcionamiento y establece una percepción inmediata del yo. Ésta es la única 
fiesta, los problemas que plantea, los métodos que se aplican a su desarrollo y las posturas que recogen las soluciones correspondientes.

De ahí que, para estudiar el método psicológico, deba seguirse un camino similar al que proseguimos en los problemas y las posturas. Así como. llegamos entonces a la conclusión de que el camino es múltiple, y a pesar de todo se reconoce una cierta unidad al hablar del problema psicológico y de la psicología misma, así también es factible concluir en la existencia de un método que se integraría por la totalidad de los métodos parciales, pues cada uno desempeña una función específica. $\mathrm{Y}$ asi como en el caso de las posturas. y problemas hablamos de una psicología sincrética que sintetiza y vincula a todas, así también se localiza un método sincrético que representa la unidad y la totalidad de los métodos parciales. Como base para determinar en qué consisten dichos métodos parciales en las posturas específicas que venimos. sosteniendo, diremos algunas palabras sobre el que llamamos método básico, fundamental o primario en su estimación dinámica.

\section{El método básico}

El método que corresponde en general a la psicologia básica, cuyo problema consiste en exponer las facultades o procesos generales del psiquismo, radica en explicar cómo se llega al conocimiento primordial de la conciencia. Dicho método procede a base de observaciones directas en la experiencia vital, sobreel flujo de la conciencia que acciona en indisoluble relación respecto a los: determinantes biológicos y las experiencias vividas, así como frente al medio. que la envuelve y los individuos que integran la colectividad.

Ese método se lleva a cabo sobre dos grandes caminos que traducen sendas posibilidades de acción, según que las observaciones se efectúen en el sujeto mismo o se proyecten en el prójimo. En cada caso se habla generalmentede un método específico; para nosotros son dos modalidades del método que designamos como básico o esencial, y consiste en observar directamente la experiencia psíquica a través de los conductos perceptivos, produciendo las. acepciones precitadas, que consisten respectivamente en la observación de uno mismo, en cuyo caso es introspección, o de los demás, dando lugar entonces a la extrospección. Sus definiciones pueden ser las siguientes:

a) La introspección o introversión es el método que consiste en observar la experiencia psicológica en nosotros.

b) La extrospección o extroversión es el método que consiste en observar la experiencia psicológica en el prójimo.

La fase interiorizante conocida como introspección o introversión es el camino metódico insustituible para percibir el flujo vivencial que se desenvuelve en nosotros mismos; capta directamente los diversos motivos de su funcionamiento y establece una percepción inmediata del yo. Ésta es la única. 
forma de aprehender con autenticidad plena el flujo vivencial de la actividad consciente que desenvuelve el psiquismo.

La acción del método introspectivo, introvertido o interiorizante, se desarrolla en paralelo a la actividad consciente del sujeto autoexaminado. Es muy probable que el hombre común se limite a vivir en el mundo, sin percatarse de lo que significa su existencia, pero a medida que evoluciona, adquiere una mayor penetración psicológica que culmina en el conocimiento de sí mismo, obtenido por medio de la introspección. Como sabemos, esta fase del método básico o directo es tenida por un método en sí, consistente en percibir los acontecimientos psicológicos que tienen verificativo en nuestra interioridad.

Por medio de dicho método se capta el flujo vivencial interno, o sea la experiencia intima donde se manifiesta el decurso vital, que en última instancia constituye el objeto único de la psicología. El empleo del propio método confiere a esta ciencia un carácter diferencial con respecto a los demás conocimientos, ya que la observación introspectiva se produce como sensación directa de lo que ocurre en nosotros mismos.

Por ejemplo, el hecho de experimentar un dolor físico proporciona al sujeto la incomparable experiencia consistente en la sensación viva y auténtica del dolor, sentido en carne propia y con una inconfundible e insustituible autenticidad. Si otra persona experimenta un dolor similar, podremos comprenderlo porque nos lo comunica verbalmente, con un relato que proporciona el conocimiento exterior de dicha sensación, pero este conocimiento no se puede comparar al que hemos experimentado en nosotros mismos.

Eventualmente, para obtener el conocimiento exterior nos auxiliamos con recursos del laboratorio, como los experimentos físicos que permiten la medición de algunas reacciones fisiológicas que se examinan en el estudio objetivo de las sensaciones. Pero el estudio externo en ningún caso reemplaza a la vivencia interna de la sensación, porque el efecto producido en la experiencia propia ofrece una imagen más vívida que cualquier relato proveniente de otra persona. Por ello, el conocimiento psicológico de los demás está sujeto en gran medida a las experiencias que hayamos vivido nosotros mismos.

El sencillo ejemplo de una sensación física como es el dolor, puede hacerse extensivo a todos los procesos psíquicos que somos capaces de experimentar, cuyo conjunto integra nada menos que la experiencia vital, es decir, el decurso de la vida misma.

El método introspectivo permite captar todos los pensamientos, sentimientos, deseos, propósitos, recuerdos y demás vivencias, es decir, toda la amplísima gama de experiencias, mediante la percepción directa del acaecer psicológico, observando en nosotros mismos la compleja dinámica que vincula estructuralmente a las vivencias percibidas, lo cual sería imposible lograr con la misma agudeza sin el concurso del método introspectivo. 
conciencia es individual e intransferible. Pero este hecho no impide producir una explicación objetiva del vivir, que llega al nivel de cientificidad cuando se efectúa conforme al canon habitual de toda ciencia, consistente en obtener leyes generales que permitan la explicación de casos particulares. Así tenemos que, siendo extremadamente singulares las vivencias cuando las experimentamos introspectivamente, el contacto con el prójimo permite llegar a generalizaciones que expresan la parte sustancial correspondiente a cada tipo de vivencia, caracterizándola en su naturaleza general y objetiva, para derivar a su explicación en la conciencia individual y subjetiva.

Por ello hemos reunido en una sola unidad metodológica dos acepciones que por regla general se distinguen y separan; el método introspectivo y el extrospectivo constituyen para nosotros dos fases inescindibles del solo método que designamos con el nombre de básico o fundamental, para expresar la función sustancial que desempeña en cuanto captación directa de la experiencia, ya sea en nosotros mismos, cual es el caso de la introspección, o en los demás, como sucede con la extrospección.

Concluyendo, el método básico supone que solamente somos capaces de reconocer lo que sucede en nuestros semejantes, en la medida en que hayamos experimentado las vivencias en nosotros mismos. De otra suerte, las reacciones que percibimos serán un signo exterior cuyo significado permanece inasequible para nosotros, desde el momento en que sólo percibimos el aspecto externo de la conducta y sus móviles íntimos nos son desconocidos.

Por ello una persona que jamás hubiese experimentado determinados sentimientos, como el amor, la ternura, la compasión, la cólera, la envidia, los celos, etc., se extrañaría profundamente al observar su producción en los demás y verificaría las reacciones externas sin comprender las causas que producen tales sentimientos, ni las modalidades diferenciales o matices que sólo puede comprender quien los experimente por el camino introspectivo y verifique comparativamente en el prójimo por el método de la extrospección. Sólo a partir de la vivencia entenderá las reacciones que se producen en los ‘demás, como semejantes a las que ha experimentado él mismo.

Tal es la esencia del método básico -introspectivo y extrospectivo- que permite obtener las observaciones primarias de la psicología, como son el conocimiento directo de nosotros mismos y de los demás. Este método sirve de base para el ulterior desarrollo de las nociones que se encuentran en la disciplina psicológica y se traduce directamente en la verificación del mecanismo básico de la conciencia, con las facultades o procesos, también básicos, que integran dicho mecanismo. 


\section{Experimental, analitico y social}

Otra de las vías metódicas que han producido grandes resultados en la investigación psicológica, es el método experimental. Como su nombre lo indica, dicho método consiste en promover los experimentos necesarios para determinar las causas y efectos del psiquismo, sus leyes constitutivas, sus variaciones concomitantes, tal como corresponde a toda disciplina científico-natural. Según criterio de algunos, la experimentación es el método más fecundo en psicología, pues por medio de la experiencia naturalista se hacen variar adecuadamente las condiciones en que se producen los hechos psíquicos.

El método experimental encuentra particular aplicación al campo de las ciencias físicas, pero también la psicología cosecha buenos frutos por su conducto, considerando que el psiquismo es en gran medida un fenómeno natural, un sistema capaz de experimentar modificaciones causadas deliberadamente; el objeto de la experimentación que se lleva a cabo en el laboratorio, tiende a observar el vínculo establecido entre la modificación de las causas y la correlativa modificación de los efectos.

Observando esta relación se formulan leyes que rigen los fenómenos psicológicos desde el punto de vista natural, o sea el que corresponde a la especie humana como parte integrante de la naturaleza, resultado de una prolongada evolución que se dilata a través de los siglos y las generaciones. La evolución biológica señala paulatinamente la integración de la conciencia, observada en la complejidad del sistema nervioso que es, entre los factores biológicos, el más influyente en la conducta, al grado de que numerosos autores de orientación biologista tienden a interpretar el comportamiento como resultado directo de la organización neuronal.

Con independencia de la razón o sinrazón que pueda asistir a semejante teoría, el hecho es que la estructura biológica del sistema nervioso, así como una serie de factores naturales, repercuten en los instintos, tendencias e inclinaciones que refleja la especie como resultado de su naturaleza, y en gran medida exponen la regularidad que puede encontrarse en el comportamiento de los individuos, gracias a lo cual se habla de leyes psicológicas, si es que puede haber leyes en psicología, pues ya sabemos que una de las características esenciales de la conducta radica en la libertad que tiene el hombre para actuar, y por consiguiente, para liberarse - hasta cierto punto- de las leyes que rigen en el campo de la naturaleza.

Pero hemos dicho "hasta cierto punto"; en esta circunscripción se contiene la más importante relativización de la libertad que disfruta y ejercita el ser humano. No olvidemos que, por muy consciente y espiritual que se le suponga, estă inmerso en la naturaleza y existe en un cuerpo biológico, sujeto a leyes naturales. Inclusive la producción de las vivencias está acompañada por diversos acontecimientos de orden físico que son susceptibles de apre- 
ciar por medio de los sistemas y métodos que rigen normalmente en la ciencia misma.

Desarrollando este sentido experimental se formaron los laboratorios de psicología a partir del siglo pasado, debido principalmente a que el auge de las ciencias naturales permitió profundizar en la comprensión de los fenómenos físicos, químicos y biológicos, a un nivel suficiente para establecer determinadas leyes basadas en la observación experimental y verificables en ella misma.

Así fue posible desenvolver una convicción establecida desde la época griega, cuando filósofos como Aristóteles y médicos como Hipócrates, llegaron a la conclusión de que la conducta y la conciencia del hombre no representan una entidad inconsútil, sino que exhiben un alto grado de materialidad por el cual es posible explicar el carácter y la personalidad del individuo, teniendo en cuenta los coeficientes biológicos que operan sobre él y contribuyendo en gran medida a comprender el sentido del comportamiento en función de las causas biológicas, según el esquema de causa a efecto que rige en los procesos naturales.

Ahí está el estudio increfblemente minucioso que se ha hecho de los mecanismos fisiológicos, de manera muy especial el sistema nervioso, que contribuye directamente a la teoría conductista de naturaleza causal; se conoce extensamente cuál es la función que desempeñan las células del sistema nervioso, las variaciones a que están sujetas, el rango de alteraciones normales y anormales, etc. De ahi deriva que, junto al estudio normal del psiquismo, se encuentra el de los casos anormales, dando origen a la psicopatología biológica, cuya expresión sistemática es la psiquiatría.

Por todo ello se comprende el papel tan importante que desempeña el estudio naturalista en la psicología moderna, al grado que su participación resulta indispensable, aunque no agota en si mismo todos los aspectos esenciales de la problemática psicológica.

El método experimental se funda en la relación biológica de la conciencia y toma de ella los elementos que considera vinculados al proceso psíquico, en cuya virtud examina la realidad psicobiológica que es base de la experimentación en esta disciplina y define cuáles son sus consecuencias en la conducta. El método que emplea es estrictamente científico y se desenvuelve con sistemas e instrumentos de laboratorio, obteniendo mediciones que suelen ser precisas, acopiando pruebas verificadoras de especie mecanicista que penetran profundamente en la organización biopsíquica, es decir, en los fenómenos biológicos que guardan relación directa con el psiquismo.

Por medio de este método experimental es posible llegar a conocimientos sumamente especializados. A través de él se explica un buen número de procesos que antiguamente permanecían ignorados e insuficientemente conocidos; la vía estricta de la experimentación arrojó luz sobre sus operaciones 
y hoy día han sido comprendidas en el estricto dominio de la ciencia, debido en gran parte al empleo del método experimental.

Continuando con nuestra exposición de los métodos que emplea la psicología en el estudio de sus problemas, encontramos el correspondiente a la psicología analítica. Puede llamarse específicamente método analítico el que desenvuelve el sistema doctrinario conocido como psicología analítica, del cual representa su instrumento metodológico.

No es común encontrar la designación de "método analítico" en psicología, y sí en cambio muy frecuente hablar de psicoanálisis, término que corresponde a un aspecto del método analítico, aunque no lo cubre en su totalidad. Efectivamente, el psicoanálisis representa el método empleado para examinar la estructura interna de la personalidad a través de la relación consciente-subconsciente y se aplica asimismo al tratamiento de algunos padecimientos psicopáticos que implican ante todo una exploración del inconsciente.

Este método se desenvuelve mediante un examen analítico, o sea distintivo de sus factores dinámicos y se lleva a cabo en la persona afectada o paciente; dicho examen lo lleva a cabo el psicólogo o terapeuta que, por desempeñar esta labor analítica, recibe también el nombre de analista. De acuerdo con la doctrina clásica, el psicoanálisis permite llegar a las motivaciones profundas de la conducta y descubrir sus verdaderas causas determinantes; se trata de un sistema efectivo para promover el análisis psicopatológico de los casos individuales.

Además del procedimiento metódico, directamente avocado al tratamiento de los casos patológicos o anormales, existe la doctrina psicoanalítica propiamente dicha que recoge la problemática de la cual deriva el método. Esta doctrina implica el método analítico normal, desarrollado según los materiales con que trabaja, de análoga manera a como sucede con las demás doctrinas psicológicas.

Dichos materiales han revelado, a través de incontables observaciones, la existencia de una dinámica estructural que relaciona los diversos aspectos de la personalidad o sean las funciones de la conciencia, como son la inconciencia, la supraconciencia y la conciencia propiamente dicha. La interrelación constante de estas funciones determina esencialmente el método de la psicología analítica, cuya aplicación práctica implica el psicoanálisis, mientras que el aspecto teórico propiamente dicho se encuentra en el método analítico.

Dicho método afirma la teoría de que todos los aspectos de la personalidad están íntimamente relacionados en virtud de una acción interconsciente por medio de la cual se vinculan los estratos dinámicos, o mejor dicho, las funciones de la personalidad: conciencia, inconciencia y supraconciencia.

Por último, la exploración sociológica de la psicología cuenta asimismo con el método correspondiente, que llamaremos método psicosocial y se distingue de los demás que emplea esta ciencia en sus variadas tareas. La dimen- 
sión colectiva de la conducta y la problemática que recoge el respectivo método de la sociopsicología adquiere extraordinaria complejidad en virtud de los numerosos elementos que actúan sobre ella, obedeciendo en igual medida a la complejidad del problema que engloba una gran cantidad de coeficientes: causales y teleológicos.

A nadie escapa la convicción de que dichos acontecimientos revisten una extraordinaria complejidad en virtud de los numerosos factores que actúan sobre ellos y que son de la más variada índole: demográficos, geográficos, etnográficos, económicos, antropológicos, históricos, psicológicos, etc., cada uno de los cuales implica un amplio sistema de conocimientos y entronca en abundante problemática derivada, que hace extraordinariamente complejo el panorama de la psicología social.

El método empleado en esta disciplina consiste fundamentalmente en descubrir las relaciones producidas entre la conducta colectiva y los coeficientes psicológicos que la determinan. Esta relación es de suyo comprensible, pues la sociedad está integrada por individuos que accionan y reaccionan conforme a determinados lineamientos básicos y generales observados por la ciencia del psiquismo. El método psicosocial mantiene esta relación como una de' sus piedras angulares, siendo otra el método general correspondiente a la sociología, que a su vez encuentra numerosas implicaciones en el vastísimo conjunto de las ciencias sociales y se traduce ulteriormente en la problemática específica de cada una.

Esta problemática acusa una estructura metodológica por cuanto los fenómenos que afloran en la sociedad son extremadamente complejos, debido al crecido número de elementos que en ellos participan y a la interacción dinámica que se manifiesta por razón de su coexistencia en la realidad psicosocial.

Esta situación fue advertida hasta fecha reciente en el terreno sociológico, pues durante mucho tiempo se creyó que la sociología era una simple acumulación de planteamientos empírico-sociales, sin reconocer la estructura orgánica que origina la interacción dinámica de tales elementos, así como la correspondiente distribución de sus problemas, en forma tal que pueda de cada uno ocuparse una disciplina especializada, contando al mismo tiempo con la proyección metodológica y formal que corresponde a la sociología propiamente dicha.

En estas condiciones, queda la sociología en calidad de una disciplina formal que representa el método orgánico de las ciencias sociales, de donde su aplicación a la psicología social es de todo punto indispensable e implica la totalidad de relaciones sociofenoménicas que se observan en la experiencia colectiva, así como la organización metodológica que se devela mediante el examen estructural y dinámico de sus relaciones.

Por medio del método psicosocial se investiga hasta qué punto el indivi- 
duo es determinable por el medio, y recíprocamente, hasta qué grado es determinable el medio por la acción de los individuos.

Este es un problema de gran importancia que se ha debatido no solamente en la disciplina que nos ocupa, sino en todo el ámbito de las ciencias sociales, llegando incluso a la filosofía de la historia por cuanto se pregunta - entre otros interrogantes - cuál es el papel que desempeña el individuo en la gestación histórica, cómo es constreñido por la sociedad y de qué manera influye él mismo sobre ella.

Este asunto presenta una doble dimensión que concierne, por una parte, a la naturaleza objetiva del problema planteado, corréspondiendo entonces a la filosofía de la historia, y por la otra se manifiesta en la proyección subjetiva, en cuyo caso pertenece directamente a la psicología social. Así observamos la trascendencia del problema, al incidir más allá de las formas sociopsiquicas del comportamiento, para ingresar en la teoría dinámica y explicativa de la historia universal.

Las conclusiones de este método se obtienen sobre el aspecto netamente psicológico de la correlación, consistente en el influjo que los estamentos sociales ejercen en la personalidad, y cómo reacciona el individuo a tal influencia para exteriorizar sus impulsos y modificar la realidad del medio en que se desenvuelve. De manera principal interesa definir el papel que desempeñan en esta gama de acciones y reacciones, los diversos sectores de la cultura que participan poderosamente en la formación del individuo, así como sus valores representativos, señalando una pauta metódica a seguir en cuanto el hombre se conduce psicosocialmente en la búsqueda de los valores culturales y la correspondiente realización de sus obras.

En efecto, la historia de la humanidad demuestra que los grupos sociológicos se encaminan a realizar los valores que reconocen implícita o explícitamente como finalidades de su comportamiento, ya sea de manera consciente, como en las culturas evolucionadas, o en forma un tanto inconsciente, como se observa en los pueblos de escaso desarrollo, dando origen a una marcada asimilación del problema sociopsíquico al dominio de la antropología social.

Puede observarse que también en este caso se verifica el principio general que hemos expuesto, consistente en la afirmación de una indisoluble analogía entre el problema planteado y el método que se emplea para resolverlo. El problema radica en las relaciones psicológicas establecidas entre individuo y sociedad; el método a seguir no podrá ser otro que la doble aplicación de las conclusiones obtenidas en la psicología individual y la sociología general, como base concreta para explicar las formas del comportamiento colectivo. Recíprocamente, la referencia establecida en las modalidades de la vida gregaria se efectúa en la sociopsicología con vías a explicar la conducta individual, auxiliándose en todo del método sociológico, que ofrece sinópticamente la explicación constitutiva y estructural de las formas de convivencia. 


\section{El método sincrético}

El desenlace de la reflexión metodológica que acometimos en paralelo a la anterior meditación sobre las posturas psicológicas, consistente en mostrar cómo y por qué cada una de ellas posee una razón que la justifica, debe ocurrir necesariamente a la concepción del método sincrético, el cual corresponde al sistema integral de la psicología, con el mismo carácter unitario que debe revestir a la psicología sincrética. En efecto, la idea de este método radica en la necesidad de incorporar los conocimientos que se obtienen sobre cada una de las direcciones psicológicas, en un solo cuerpo de doctrina, capaz de responder a la pregunta capital que se presenta en el estudio de la psicologia: ¿qué es la conciencia?

Por lo que hemos dicho, cada una de tales direcciones contesta al mismo interrogante en función del punto de vista que le es privativo $y$, por consiguiente, resulta parcial con respecto a la totalidad obtenible al unificar las grandes vertientes psicológicas. En la construcción de dicha unidad, la respuesta que otorga la psicología básica al exponer las categorías psíquicas en cuanto facultades y procesos de la conciencia, debe complementarse en la convicción que cada uno de tales elementos - considéresele como facultad, categoría o proceso- es sólo un componente de la estructura conciencial básica, cuya totalidad implica una serie de relaciones intraconscientes, tal como lo enseña la psicología moderna, especialmente en su modalidad analítica. Esta psicología reconoce asimismo la estrecha correlación de los factores biológicos, según lo comprueba la tesis naturalista, y, por último, admite la interrelación que establecen los individuos en el seno de la colectividad, como pone de relieve la psicología social.

Cada una de tales posturas exhibe la propia justificación en la medida en que sus especialidades fundan la relación en el problema integral de la conciencia, que a su vez se traduce en la conducta, el temperamento, el carácter y la personalidad, implicando a través de esta relación la serie de elementos doctrinarios que en su respectivo sistema de investigaciones arrojan luz sobre la interpretación que confieren al ser consciente las posturas específicas.

Pero esta relación no basta para desentrañar integralmente el problema; se requiere además una procuración conjunta de las vertientes psicológicas, que haga confluir su caudal en el receptáculo común que designamos como psicología sincrética, cuyo propósito es obtener el conocimiento integral de la conciencia y cuyo método se desenvuelve en el descubrimiento de las relaciones que surgen de la interinfluencia reciproca que presenta el hombre en el comportamiento. La ciencia descubre e interpreta esa interinfluencia a través de sus diferentes proyecciones, produciendo una perspectiva mucho más amplia que cualquiera de las posturas específicas, debido a que no solamente engloba el rendimiento particular que se encuentra en todas ellas, sino 
que aborda también la cuestión crucial de establecer las relaciones que se manifiestan en los diversos aspectos del ser humano y que obviamente no pueden ser comprendidos en ninguna de las posturas particulares que, al radicalizarse, conviértense en unilaterales.

Como puede observarse, la psicología sincrética no constituye una vertiente adicional, del mismo tipo que observamos en la psicología básica, biológica, analítica o social, cada una de las cuales asimila una forma específica de relación entre la conciencia y un sistema determinativo que se proyecta en ella. El método sincrético no tiene como objeto ningún aspecto particular del psiquismo, sino la estructura total, observada inicialmente a través de las proyecciones especificas tantas veces mencionadas. La tarea sincrética se desenvuelve en un establecimiento diferencial de relaciones en las vertientes del saber psicológico, con lo cual no se comete ninguna arbitrariedad abstractiva, sino que se prosigue en la natural determinación de las vinculaciones que manifiesta el mecanismo psicológico.

No será, en efecto, una mera invención buscar los vínculos entre el problema analítico y el biológico, y de ambos con el sociológico, que a su vez deberán fundar sus reflexiones en los principios generales de la psicología básica. Todo ello corresponde dinámicamente a una realidad que es ella misma dinámica y relacionante, cual se observa en el funcionamiento concreto de la conciencia; de ningún modo se manifiesta con el sentido monotónico que le confieren pretendidamente dichas posturas en su interpretación unilateral, sino a través de la interacción conjunta representada fenoménicamente por la acción empiriológica de la conducta. Es cierto que el procedimiento abstractivo resulta indispensable para fundar los aspectos particulares de la investigación, y como tal constituye el inicio de toda pesquisa, pero paralelamente al desmembramiento analítico de los diferentes aspectos del comportamiento debe producirse la unidad sincrética que realiza y al mismo tiempo explica la verdadera estructura de la personalidad.

Miguel Bueno

Instituto de InVESTIGACiones Filosóficas

Universidad Nacional Autónoma de México 